\title{
SSR-based association mapping of salt tolerance in cotton (Gossypium hirsutum L.)
}

\author{
Y.L. Zhao, H.M. Wang, B.X. Shao, W. Chen, Z.J. Guo, H.Y. Gong, \\ X.H. Sang, J.J. Wang and W.W. Ye \\ Anyang, Henan, China \\ Corresponding author: H.M. Wang \\ E-mail: aywhm@163.com / yunleizhao2002@126.com \\ Genet. Mol. Res. 15 (2): gmr. 15027370 \\ Received August 5, 2015 \\ Accepted January 4, 2016 \\ Published May 25, 2016 \\ DOI http://dx.doi.org/10.4238/gmr.15027370
}

State Key Laboratory of Cotton Biology, Institute of Cotton Research of CAAS,

\begin{abstract}
The identification of simple sequence repeat (SSR) markers associated with salt tolerance in cotton contributes to molecular assisted selection (MAS), which can improve the efficiency of traditional breeding. In this study, 134 samples of upland cotton cultivars were selected. The seedling emergence rates were tested under $0.3 \% \mathrm{NaCl}$ stress. A total of 74 SSR markers were used to scan the genomes of these samples. To identify SSR markers associated with salt tolerance, an association analysis was performed between salt tolerance and SSR markers using TASSEL 2.1, based on the analysis of genetic structure using Structure 2.3.4. The results showed that the seedling emergence rates of 134 cultivars were significantly different, and 27 salt-sensitive and 10 salt-tolerant cultivars were identified. A total of 148 loci were found in 74 SSR markers involving 246 allelic variations, which ranged from 2 to 7 with an average of 3.32 per SSR marker. The gene diversity ranged from 0.0295 to 0.4959 , with the average being 0.2897 . The polymorphic information content ranged from0.0290 to 0.3729 , with the average being 0.2381 . This natural population was classified into two subgroups by Structure 2.3.4, containing 89 and 45 samples, respectively. Finally, eight SSR sites associated with salt tolerance ware
\end{abstract}


found through an association analysis, with the rate of explanation ranging from 2.91 to $7.82 \%$ and an average of $4.32 \%$. These results provide reference data for the use MAS for salt tolerance in cotton.

Key words: Upland cotton; Associate analysis; SSR molecular markers; Salt tolerance

\section{INTRODUCTION}

Soil salinity and alkalinity are important abiotic stresses that affect crop productivity (Li et al., 2012). Globally, around $20 \%$ of irrigated agricultural land has been reported to be affected by salinity and alkalinity (Shi, 2004), with the proportion in China being higher than the global average (Lin and Dilbar, 2007). Utilization of soil salinity and alkalinity has become an important objective for scientists. As a naturally salt tolerant crop, cotton is regarded as an ideal pioneer for the utilization of soil salinity and alkalinity. Furthermore, the breeding of salt tolerant cultivars is important for improving cotton yields in fields with high soil salinity and alkalinity (Zhang et al., 2011).

With rapid developments in the fields of molecular biology and biotechnology, especially with the exploitation of molecular markers, exploring quantitative traits of plants by association mapping has become a focus of plant genome research. Association mapping, which is based on linkage disequilibrium (LD), has been used to investigate the relationships between target traits and molecular markers or candidate genes in maize, rice, soybean, and cotton (Zhu et al., 2008). Thirunavukkarasu et al. (2014) studied the functional mechanisms of drought tolerance in subtropical maize using genome-wide association mapping, and found that single nucleotide polymorphisms (SNPs) associated with ABA-dependent signaling pathways played a major role in the plant's response to stress by regulating a series of functions including flowering, root development, auxin metabolism, guard cell functions, and the scavenging of reactive oxygen species. Kumar et al. (2015) implemented GenomeWide Association Mapping (GWAS) to identify loci controlling salinity tolerance in rice, and reported 60 SNPs (loci) to be significantly associated with the $\mathrm{Na}^{+} / \mathrm{K}^{+}$ratio and other traits observed under stress conditions. In soybean, molecular markers associated with seed germination under salt stress were detected by genome-wide association analysis, resulting in the detection of eight SNPs or suggestive SNPs that were co-associated with two salt tolerance indices (Kan et al., 2015). Zhao et al. (2014) genotyped 158 elite cotton germplasms using 212 simple sequence repeat (SSR) markers and studied genetic structure and linkage disequilibrium (LD), before performing association mapping for Verticillium wilt resistance. This resulted in the detection of 42 loci that were significantly associated with Verticillium wilt resistance in cotton. Despite this, to date, no studies have identified genes associated with salinity tolerance using SSR marker-based association mapping in cotton. This study aimed to identify SSR markers associated with salt tolerance at the seedling stage in diverse cotton accessions using association analysis. The objectives of the study were i) to analyze the genetic variations of traits contributing to salt tolerance at the seedling stage, ii) to analyze the genetic structure of a diverse cotton collection, and, iii) to identify SSRs and possible candidate genes underlying salt tolerance at the seedling stage in cotton. The major SSR loci and possible candidate genes detected in this study may be useful for the genetic improvement of cotton and will provide a basis for marker assisted selection (MAS) for salt tolerance in cotton. 


\section{MATERIAL AND METHODS}

\section{Plant materials}

A total of 134 cotton (Gossypium hirsutum L.) cultivars were selected for this study, including intermediate breeding materials (62), main cultivars of different cotton-growing regions in China (48), transgenic materials (22), and cultivars from America (3). A salt-tolerant cultivar (Zhong 07) and a salt-sensitive cultivar (Zhong S9612) were used as controls for the estimation of salt tolerance.

\section{Phenotypic evaluation}

Salt tolerance was evaluated using the method of sand culture. Culture medium was created using the following procedure: sodium chloride $(\mathrm{NaCl})$, high temperature-sterilized sand and sterile water were mixed thoroughly, with a $\mathrm{NaCl}$ content of $0.3 \%$ by weight of the sand and a water content of $22 \%$ by the total weight of $\mathrm{NaCl}$ and sand. Seeds of different cultivars were planted in germinating boxes containing aliquots of culture medium under conditions of 14-h light/day, and day and night temperatures of $28^{\circ}$ and $25^{\circ} \mathrm{C}$, respectively. The experimental design was a randomized block with three replicates. After two weeks, the emergence rate was estimated for each sample. ANOVA was performed to compare the emergence rate of 134 cultivars by SAS 9.2 software.

\section{Isolation of plant DNA}

DNA was isolated from cotton seedling leaves by means of a cetyltrimethylammonium bromide (CTAB) procedure (Paterson et al., 1993). Each PCR reaction was carried out in a volume of $10 \mu \mathrm{L}$ containing $1 \mathrm{X}$ PCR buffer, $1.5 \mathrm{mM} \mathrm{MgCl}_{2}, 0.20 \mathrm{mM}$ dNTPs, $2.5 \mu \mathrm{M}$ each of upstream and downstream primers, and $0.5 \mathrm{U}$ Taq polymerase. PCR conditions were as follows: $95^{\circ} \mathrm{C}$ initial denaturation for $3 \mathrm{~min} ; 94^{\circ} \mathrm{C}$ for $30 \mathrm{~s}, 56^{\circ} \mathrm{C}$ for $90 \mathrm{~s}$, and $72^{\circ} \mathrm{C}$ for $1 \mathrm{~min}$ for 30 cycles followed by an extension at $72^{\circ} \mathrm{C}$ for $5 \mathrm{~min}$. PCR products were analyzed by $10 \%$ polyacrylamide gel electrophoresis. The gels were silver-stained to visual differential bands among different samples (Zhang et al., 2000).

\section{Statistical analysis}

The population structure of 134 cultivars was estimated using the model-based (Bayesian) cluster software STRUCTURE 2.3.4 (Pritchard and Wen, 2007). STRUCTURE was run under the 'admixture model' with a burn-in period of 10,000 followed by 100,000 replications of Markov Chain Monte Carlo. Five independent runs were performed with the number of clusters $(\mathrm{K})$ varying from 1 to 10 . An ad hoc measure, $D k$, based on the relative rate of change in the likelihood of the data between successive $\mathrm{K}$ values was used to determine the optimal number of clusters (Evanno et al., 2005). Marker-trait association was estimated using the general linear model (GLM) of the TASSEL 2.1 software package (Bradbury et al., 2007), in which the percentage of admixture of each accession (Q matrix) was used as a covariate to conduct the regression between phenotypic variation and markers. 


\section{RESULTS}

\section{Phenotypic analysis of salt tolerance}

Some salt-tolerant and salt-sensitive cultivars were detected by estimating the emergence rate of 134 cultivars under $0.3 \% \mathrm{NaCl}$ stress (Table 1). The frequency distribution histogram was produced according to the emergence rate of 134 cultivars (Figure 1). The emergence rate of most cultivars was in the range of 60 to $80 \%$. The salt-sensitive cultivar Zhong S9612 had an emergence rate of $37.67 \%$, and the salt-tolerant cultivar Zhong 07 had an emergence rate of $84.34 \%$. A total of 27 cultivars were regarded as salt-sensitive and had lower emergence rates than the salt-sensitive cultivar Zhong S9612, and 10 cultivars were regarded as salt-tolerant and had higher emergence rates than the salt-tolerant cultivar Zhong 07 (Table 1).

Table 1. Salt tolerant and salt sensitive materials screened during the preliminary selection.

\begin{tabular}{|c|c|c|c|}
\hline Salt-sensitive material & Seedling emergence rate (\%) & Salt-tolerant material & Seedling emergence rate (\%) \\
\hline $2010 \mathrm{yc}-2$ & 0.47 & ZhongZZS-31 & 84.44 \\
\hline ZongZZS-29 & 0.67 & Yongmian2 & 84.54 \\
\hline YNB-1 & 3.08 & ZhongZZS-30 & 87.92 \\
\hline Jinke178 & 3.33 & $\mathrm{~T}-1$ & 88.00 \\
\hline ZhongZZS-36 & 3.79 & ZhongZZS-34 & 88.26 \\
\hline ZhongZZS-45 & 4.50 & ZhongZZS-42 & 89.05 \\
\hline ZhongZZS-22 & 5.50 & ZhongZZS-4 & 89.74 \\
\hline 80619 & 10.00 & ZhongZZS-15 & 92.50 \\
\hline 50217 & 11.23 & Hebeikanghuang & 92.86 \\
\hline Wuzhuan5 & 12.75 & Zhong3538 & 93.98 \\
\hline ZhongZZS-14 & 13.00 & & \\
\hline Wuzhuan4 & 14.23 & & \\
\hline ZhongZZS-18 & 14.35 & & \\
\hline Xinluzhong36 & 17.00 & & \\
\hline Han109 & 17.00 & & \\
\hline 2010BP4 & 17.50 & & \\
\hline Arcot-1 & 21.11 & & \\
\hline Nongdamian8 & 29.00 & & \\
\hline Xinqiumian1 & 30.38 & & \\
\hline ZhongZZS-19 & 30.50 & & \\
\hline Difen765078 & 30.67 & & \\
\hline ZhongZZS-47 & 34.00 & & \\
\hline GK99-1 & 35.00 & & \\
\hline 2010BP5 & 35.90 & & \\
\hline Zhong990659 & 36.50 & & \\
\hline Wuzhuan2 & 37.00 & & \\
\hline Jimian228 & 37.00 & & \\
\hline
\end{tabular}

ANOVA showed that there were significant differences $(\mathrm{P}<0.01)$ for the estimated emergence rate among the 134 cultivars (Table 2), implying that sufficient phenotypic polymorphism existed in this group to make it suitable for association mapping.

\section{Genetic diversity}

Allele number, gene diversity, and polymorphism information content (PIC) were calculated to estimate genetic diversity within the group (Table 3 ). The gene diversity and PIC 
were $0.2897(0.0295-0.4959)$ and $0.2381(0.0290-0.3729)$, respectively. The number of alleles for 74 SSR markers was in the range of 2-7, of which the markers BNL3823and TMB1296 were the most abundant. The total number of polymorphic SSR loci in 134 cultivars was 148, involved in 246 alleles, with an average of 3.32 alleles per locus.

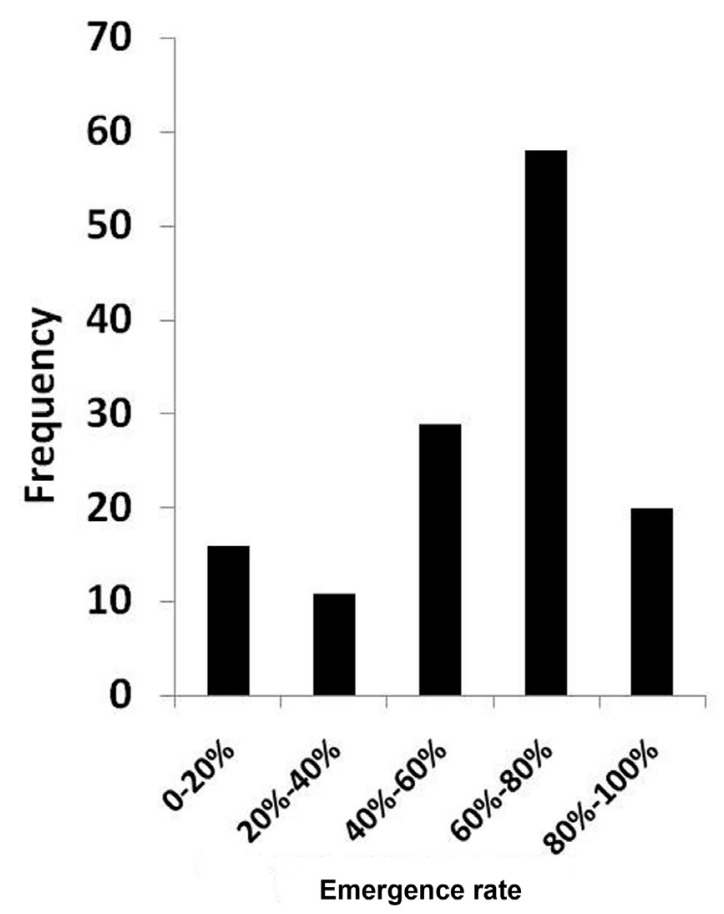

Figure 1. Frequency distribution diagram of the emergence rate of seedlings of 134 upland cotton cultivars under the $0.3 \% \mathrm{NaCl}$ stress.

Table 2. ANOVA of the seedling emergence rate of 134 upland cotton cultivars under $0.3 \% \mathrm{NaCl}$ stress.

\begin{tabular}{l|c|c|c|c|c}
\hline Source & d.f. & Squares & Mean square & F value & $\operatorname{Pr}>\mathrm{F}$ \\
\hline Model & 133 & 18.03 & 0.136 & 10.46 & $<0.0001$ \\
\hline Error & 225 & 2.915 & 0.013 & & \\
\hline Corrected total & 358 & 20.95 & & & \\
\hline
\end{tabular}

\section{Population structure of 134 cotton cultivars}

Inference of the population structure of the 134 cotton cultivars was performed using the model-based software STRUCTURE. The LnP(D) value increased continuously with $\mathrm{K}$ from 1 to 10 (Figure 2A), and the highest $\Delta K$ value was observed at $\mathrm{K}=2$ followed by a drastic decline of $\Delta K$ from $\mathrm{K}=3$ (Figure 2B). Therefore, this group could be divided into two subgroups (Figure 3). Using a probability of membership threshold of 50\%, 89 and 45 lines were assigned into the two subgroups, respectively. 
Table 3. Diversity of 74 simple sequence repeat (SSR) markers.

\begin{tabular}{|c|c|c|c|c|}
\hline Marker & Chromosome & Gene diversity & PIC* & No. of alleles \\
\hline NAU5120 & Chr16 & 0.4368 & 0.3410 & 4 \\
\hline NAU5152 & Chr7/16 & 0.1752 & 0.1599 & 2 \\
\hline NAU5428 & Chr11 & 0.4889 & 0.3694 & 2 \\
\hline TMB1767 & Chr13/18 & 0.1056 & 0.1000 & 4 \\
\hline BNL2733 & Chr7 & 0.1988 & 0.1791 & 3 \\
\hline NAU2277 & Chr2 & 0.4469 & 0.3465 & 6 \\
\hline HAU0119 & Chr17 & 0.3427 & 0.2837 & 4 \\
\hline DPL0209 & Chr11 & 0.3017 & 0.2555 & 2 \\
\hline DPL0212 & Chr19 & 0.4959 & 0.3729 & 2 \\
\hline NAU2627 & Chr16 & 0.0927 & 0.0882 & 3 \\
\hline DPL0249 & Chr18 & 0.2439 & 0.2136 & 2 \\
\hline NAU2820 & Chr7/16 & 0.4318 & 0.3381 & 4 \\
\hline NAU2894 & Chr19 & 0.2647 & 0.2238 & 3 \\
\hline NAU2954 & Chr23/25 & 0.1000 & 0.0946 & 2 \\
\hline NAU3017 & Chr13/18 & 0.3516 & 0.2887 & 4 \\
\hline NAU3096 & Chr5/19 & 0.3420 & 0.2831 & 3 \\
\hline NAU3100 & Chr23 & 0.4839 & 0.3667 & 4 \\
\hline NAU3201 & Chr8/24 & 0.0508 & 0.0495 & 4 \\
\hline NAU3212 & Chr5 & 0.3168 & 0.2658 & 4 \\
\hline NAU3414 & Chr9/23 & 0.2902 & 0.2478 & 2 \\
\hline NAU3419 & Chr2 & 0.4500 & 0.3483 & 3 \\
\hline NAU3468 & Chr13 & 0.3516 & 0.2887 & 4 \\
\hline NAU3486 & Chr16 & 0.0437 & 0.0426 & 4 \\
\hline NAU3609 & Chr19 & 0.3177 & 0.2671 & 4 \\
\hline NAU3820 & Chr14 & 0.3669 & 0.2850 & 4 \\
\hline NAU4031 & Chr5 & 0.2275 & 0.2015 & 4 \\
\hline NAU4042 & Chr19 & 0.0295 & 0.0290 & 3 \\
\hline NAU5064 & Chr11 & 0.2633 & 0.2282 & 4 \\
\hline NAU5107 & Chr1 & 0.1315 & 0.1227 & 3 \\
\hline NAU5163 & Chr1 & 0.1118 & 0.1052 & 3 \\
\hline NAU5233 & Chr3 & 0.1759 & 0.1526 & 3 \\
\hline NAU5351 & Chr10/11/13 & 0.4219 & 0.3325 & 3 \\
\hline NAU5433 & Chr6 & 0.3661 & 0.2987 & 4 \\
\hline NAU5463 & Non & 0.3288 & 0.2741 & 3 \\
\hline NAU5467 & Chr14 & 0.3618 & 0.2958 & 2 \\
\hline BNL1026 & Chr7/16 & 0.1898 & 0.1712 & 2 \\
\hline BNL1040 & Chr18 & 0.1056 & 0.1000 & 4 \\
\hline BNL2766 & $\mathrm{Chr} 7 / 16$ & 0.0854 & 0.0817 & 2 \\
\hline CIR0253 & Chr5/22 & 0.2270 & 0.2009 & 2 \\
\hline CIR0328 & Chr5 & 0.4930 & 0.3715 & 4 \\
\hline JESPR204 & Chr5/13/18 & 0.0724 & 0.0697 & 4 \\
\hline NAU1043 & Chr7 & 0.3513 & 0.2701 & 3 \\
\hline NAU2121 & Chr5 & 0.4918 & 0.3709 & 4 \\
\hline NAU2274 & Chr5/19 & 0.3420 & 0.2831 & 3 \\
\hline NAU2980 & Chr18 & 0.1506 & 0.1392 & 3 \\
\hline CIR0412 & Chr7 & 0.1062 & 0.0980 & 4 \\
\hline NAU2265 & Chr2 & 0.4485 & 0.4485 & 4 \\
\hline NAU3424 & Chr16 & 0.1087 & 0.1087 & 3 \\
\hline NAU3703 & Chr11 & 0.2687 & 0.2687 & 4 \\
\hline JESPR292 & Chr16 & 0.1752 & 0.1599 & 2 \\
\hline NAU3839 & Chr3 & 0.3410 & 0.2829 & 2 \\
\hline BNL3295 & Chr6 & 0.0718 & 0.0693 & 4 \\
\hline BNL3414 & Chr12 & 0.1253 & 0.1175 & 2 \\
\hline NAU1369 & Chr24/25 & 0.3082 & 0.2599 & 4 \\
\hline NAU2723 & Chr9 & 0.4554 & 0.3517 & 2 \\
\hline NAU2580 & Chr6/25 & 0.4627 & 0.3556 & 4 \\
\hline BNL3469 & Non & 0.1877 & 0.1642 & 6 \\
\hline BNL3823 & Chr23 & 0.0448 & 0.0438 & 7 \\
\hline BNL3971 & Chr2/17 & 0.4224 & 0.3332 & 4 \\
\hline
\end{tabular}

Continued on next page 
Table 3. Contributed.

\begin{tabular}{|c|c|c|c|c|}
\hline Marker & Chromosome & Gene diversity & PIC* & No. of alleles \\
\hline BNL3988 & Chr4 & 0.2726 & 0.2354 & 2 \\
\hline BNL3255 & Chr8/4 & 0.2777 & 0.2376 & 2 \\
\hline BNL3452 & Chr19 & 0.2293 & 0.2026 & 3 \\
\hline BNL1053 & Chr11/21 & 0.4890 & 0.3694 & 4 \\
\hline TMB1296 & Chr19/25 & 0.3979 & 0.3127 & 7 \\
\hline NAU0980 & Chr11 & 0.2617 & 0.2271 & 3 \\
\hline NAU1047 & Chr23 & 0.4462 & 0.3464 & 3 \\
\hline NAU1102 & Chr19 & 0.4178 & 0.3301 & 4 \\
\hline SWU10056 & Chr16 & 0.2894 & 0.2282 & 4 \\
\hline SWU10161 & Chr16 & 0.4298 & 0.3369 & 2 \\
\hline SWU10214 & Chr16 & 0.4264 & 0.3349 & 2 \\
\hline SWU10054 & Chr16 & 0.2844 & 0.2253 & 4 \\
\hline SWU10163 & Chr16 & 0.4351 & 0.3400 & 4 \\
\hline JESPR274 & Chr9/23/26 & 0.3407 & 0.2606 & 2 \\
\hline SWU10218 & Chr16 & 0.4945 & 0.3722 & 2 \\
\hline
\end{tabular}

$\mathrm{PIC}=$ polymorphism information content.
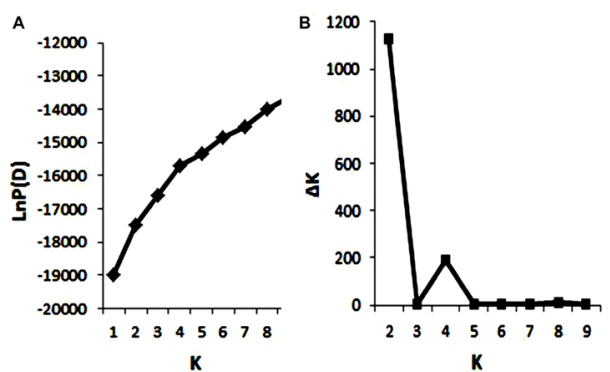

Figure 2. Line chart of $\mathrm{K}$ (the number of clusters) with $\operatorname{LnP}(\mathrm{D})$ (the log likelihood of the data) and $\Delta K$ [an ad hoc statistic based on the second-order rate of change in $\mathrm{LnP}(\mathrm{D})$ between successive $\mathrm{K}$ values].

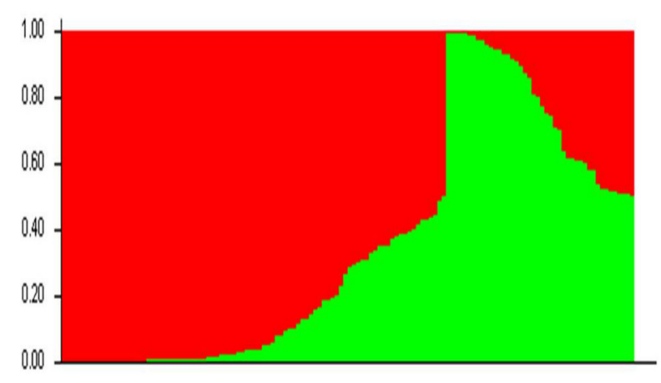

Figure 3. Population structure of 134 upland cotton cultivars. Numbers beside the vertical axis represent the probability of membership, and the two subgroups are represented by red and green, respectively.

\section{Association analysis of SSR markers with salt tolerance}

Associations between 74 marker loci and salt tolerance were determined by the GLM method. Eight marker loci with the phenotypic variation range of 2.91 to $7.82 \%$ were found to be significantly associated with salt tolerance in cotton (Table 4). Among these eight marker loci, two (NAU2580-1 and NAU2580-2) were highly significantly associated with salt tolerance and explained more phenotypic variation (7.82 and 6.26\%, respectively) than other loci. 
Table 4. Loci associated with traits and their explained phenotypic variation.

\begin{tabular}{l|c|c|c}
\hline Marker & Chromosome & P value & Explained phenotypic variation (\%) \\
\hline NAU2580-1** & Chr6/25 & 0.001 & 7.82 \\
\hline NAU2580-2** & Chr6/25 & 0.004 & 6.26 \\
\hline BNL3414* & Chr9/23 & 0.014 & 4.50 \\
\hline HAU0119-1* & Chr17 & 0.029 & 3.56 \\
\hline SWU10054-1* & Chr16 & 0.038 & 3.23 \\
\hline TMB1296-1* & Chr19 & 0.039 & 3.20 \\
\hline NAU3100-1* & Chr23 & 0.043 & 3.10 \\
\hline NAU3486-2* & Chr16 & 0.049 & 2.91 \\
\hline
\end{tabular}

*and **Indicate a significant correlation at $\mathrm{P}<0.05$ and $\mathrm{P}<0.01$, respectively.

\section{DISCUSSION}

Salt tolerance in cotton is very complex, as shown by Shen et al. (2001), who studied the inheritance of salt tolerance using Hayman's diallel cross analysis. Those authors suggested that both additive and dominant effects appeared to be important for the expression of variation under salt stress, and that the effect of genes with additive properties was more pronounced. For a long time, the focus of cotton breeding has been on improving the yield, fiber quality, and resistance to disease and pests, and few studies have investigated the improvement of salt tolerance in cotton. It is important to collect salt tolerant germplasm resources in order to enrich the genetic variation in cultivated salt tolerance cotton varieties. In this study, 134 cotton cultivars were used to estimate salt tolerance in an incubator under stable test conditions. The results showed that 27 cultivars could be regarded as salt-sensitive and 10 could be regarded as salt-tolerant, which provides a reference for cotton salt tolerance breeding. ANOVA showed that significant differences exist in phenotypes of salt tolerance among the 134 cultivars, implying that sufficient phenotypic variation exists in this group, thus making it suitable for association mapping.

In traditional breeding, it is very inefficient to breed varieties with salt tolerance by hybridizing different salt-tolerance lines. By screening molecular markers that are related to salt tolerance, and combining molecular and traditional breeding, the efficiency of salt tolerant breeding in cotton can be greatly improved. Recently, Zhang et al. (2010) screened two salttolerant cotton accessions, G. hirsutum L. cv. CCRI35 and G. hirsutum L. Zhong07, and two salt-sensitive cotton accessions, G. hirsutum L. cv. CCRI12 and G. hirsutum L. Xinyan96-48, with 274 SSR markers, and subsequently identified 10 primer pairs that could be used to detect salt-tolerance in cotton. Wang et al. (2014) developed a total of 132 pairs of non-redundant expressed sequence tag-simple sequence repeat (EST-SSR) primers related to salt-tolerance according to salt-resistant cotton ESTs and performed genetic diversity and evolution analyses on cotton. To some extent, these studies have accelerated the application of molecular markers to the breeding of salt-tolerance in cotton.

Two measures, quantitative trait loci (QTL) mapping and association mapping, are usually used to detect molecular markers associated with yield, quality, and resistance. In contrast to QTL mapping, which is based on bi-parental populations, association mapping is based on LD and uses a sample of lines from the broader breeding population that are unrelated to any specific crossing design (Zhu et al., 2008). Therefore, a higher number of historical recombination events can be explored in natural populations than in the bi-parental segregating populations, resulting in a higher resolution of QTL mapping (Ersoz et al., 2007). 
In this study, association mapping was conducted to estimate marker-trait association using the GLM in the TASSEL 2.1 software package. False association was avoided by estimating population structure and proceeding the regression between the phenotypic variation and markers with the percentages of admixture of each accession ( $Q$ matrix) as covariates. A total of eight marker loci associated with salt tolerance in cotton were identified, among which two loci (NAU2580-1and NAU2580-2) explained the most phenotypic variation of salt tolerance, implying a strong association between the marker NAU2580 and salt tolerance genes in cotton. This paper tentatively explored the associations between SSR markers and salt tolerance in cotton, and the results provide references for MAS of salt tolerance in cotton.

\section{Conflicts of interest}

The authors declare no conflict of interest.

\section{ACKNOWLEDGMENTS}

Research supported by the National Hi-Tech Research and Development Program of China (Grant \#2012AA101108-02-02) and by the Basic R\&D Operation Special Fund for the Central Level, Non-Profit, Scientific Research Institutes, China (\#1610162015A02). The funders had no role in the study design, data collection, analysis, decision to publish, or preparation of the manuscript.

\section{REFERENCES}

Bradbury PJ, Zhang Z, Kroon DE, Casstevens TM, et al. (2007). TASSEL: software for association mapping of complex traits in diverse samples. Bioinformatics 23: 2633-2635. http://dx.doi.org/10.1093/bioinformatics/btm308

Ersoz ES, Yu J and Buckler ES (2007). Applications of linkage disequilibrium and association mapping in crop plants. In: Genomics-assisted crop improvement-Vol 1: Genomics approaches and platforms (Varshney RK and Tuberosa R, eds.).Springer, Dordrecht, 97-120

Evanno G, Regnaut S and Goudet J (2005). Detecting the number of clusters of individuals using the software STRUCTURE: a simulation study. Mol. Ecol. 14: 2611-2620. http://dx.doi.org/10.1111/j.1365-294X.2005.02553.x

Kan G, Zhang W, Yang W, Ma D, et al. (2015). Association mapping of soybean seed germination under salt stress. Mol. Genet. Genomics 290: 2147-2162.http://dx.doi.org/10.1007/s00438-015-1066-y

Kumar V, Singh A, Mithra SVA, Krishnamurthy SL, et al. (2015). Genome-wide association mapping of salinity tolerance in rice (Oryza sativa). DNA Res. 22: 133-145.http://dx.doi.org/10.1093/dnares/dsu046

Li J, Pu L, Zhu M and Zhang R (2012). The status of soil salinization and future research focus. Acta Geogr. Sin. 67: 1233-1245.

Lin J and Dilbar S (2007). Progress in the study on soil salinization. J. Xinjiang Univ. (Nat. Sci. Ed.) 24: 318-323.

Paterson AH, Brubaker CL and Wendel JF (1993). A rapid method for extraction of cotton (Gossypiumspp) genomic DNA suitable for RFLP and PCR analysis. Plant Mol. Biol. Rep. 11: 122-127. http://dx.doi.org/10.1007/BF02670470

Pritchard JK and Wen W (2007). Documentation for STRUCTURE Software: Version 2.2. The University of Chicago Press, Chicago.

Shen F, Yu Y, Bi J, Liu F, et al. (2001). A diallel analysis of salt tolerance in upland cotton. Acta Agron. Sin. 27: 50-54.

Shi Y (2004). Research of land desertification and soil and water resources in the northwest area. China Sciences Press, Peking.

Thirunavukkarasu N, Hossain F, Arora K, Sharma R, et al. (2014). Functional mechanisms of drought tolerance in subtropical maize (Zea mays L.) identified using genome-wide association mapping. BMC Genomics 15: 1182. http:// dx.doi.org/10.1186/1471-2164-15-1182

Wang BH, Zhu P, Yuan YL, Wang CB, et al. (2014). Development of EST-SSR markers related to salt tolerance and their application in genetic diversity and evolution analysis in Gossypium. Genet. Mol. Res. 13: 3732-3746. http://dx.doi. org/10.4238/2014.May.13.1 
Zhang GW, Lu HL, Zhang L, Chen BL, et al. (2011). Salt tolerance evaluation of cotton (Gossypium hirsutum) at its germinating and seedling stages and selection of related indices. Ying Yong Sheng Tai Xue Bao 22: 2045-2053.

Zhang J, Wu Y, Guo W and Zhang T (2000). Fast screening of microsatellite markers in cotton with PAGE/silver staining. J. Cotton Sci. 12: 267-269.

Zhang L, Ye W, Wang J and Fang B (2010). Studies of Salinity-tolerance with SSR Markers on G. hirsutum L. J. Cotton Sci. 22: $175-180$.

Zhao Y, Wang H, Chen W and Li Y (2014). Genetic structure, linkage disequilibrium and association mapping of Verticillium wilt resistance in elite cotton (Gossypium hirsutum L.) germplasm population. PLoS One 9: e86308. http://dx.doi.org/10.1371/journal.pone.0086308

Zhu C, Gore M, Buckler ES and Yu J (2008). Status and prospects of association mapping in plants. Plant Genome 1: 5-20. http://dx.doi.org/10.3835/plantgenome2008.02.0089 